\title{
All-Atom Molecular Dynamics Elucidating Molecular Mechanisms of Single-Transmembrane Model Peptide \\ Dimerization in a Lipid Bilayer.
}

Hayato Itaya ${ }^{1}$, Kota Kasahara ${ }^{2, *}$, Qilin $\mathrm{Xie}^{3}$, Yoshiaki Yano ${ }^{4, \dagger}$, Katsumi Matsuzaki ${ }^{4}$, Takuya Takahashi ${ }^{2}$.

1. Graduate School of Life Sciences, Ritsumeikan University, 1-1-1 Noji-Higashi, Kusatsu, Shiga 5258577, Japan

2. College of Life Sciences, Ritsumeikan University, 1-1-1 Noji-Higashi, Kusatsu, Shiga 525-8577, Japan

3. College of Pharmaceutical Sciences, Ritsumeikan University, 1-1-1 Noji-Higashi, Kusatsu, Shiga 525-8577, Japan

4. Graduate School of Pharmaceutical Sciences, Kyoto University, 46-29 Yoshida-Shimo-Adachi-cho, Sakyo-ku, Kyoto 606-8501, Japan

*To whom correspondence should be addressed: Kota Kasahara, ktkshr@fc.ritsumei.ac.jp

† Present address: School of Pharmaceutical Sciences, Mukogawa Women’s University, 11-68 Koshien Kyuban-cho, Nishinomiya, Hyogo 663-8179, Japan 


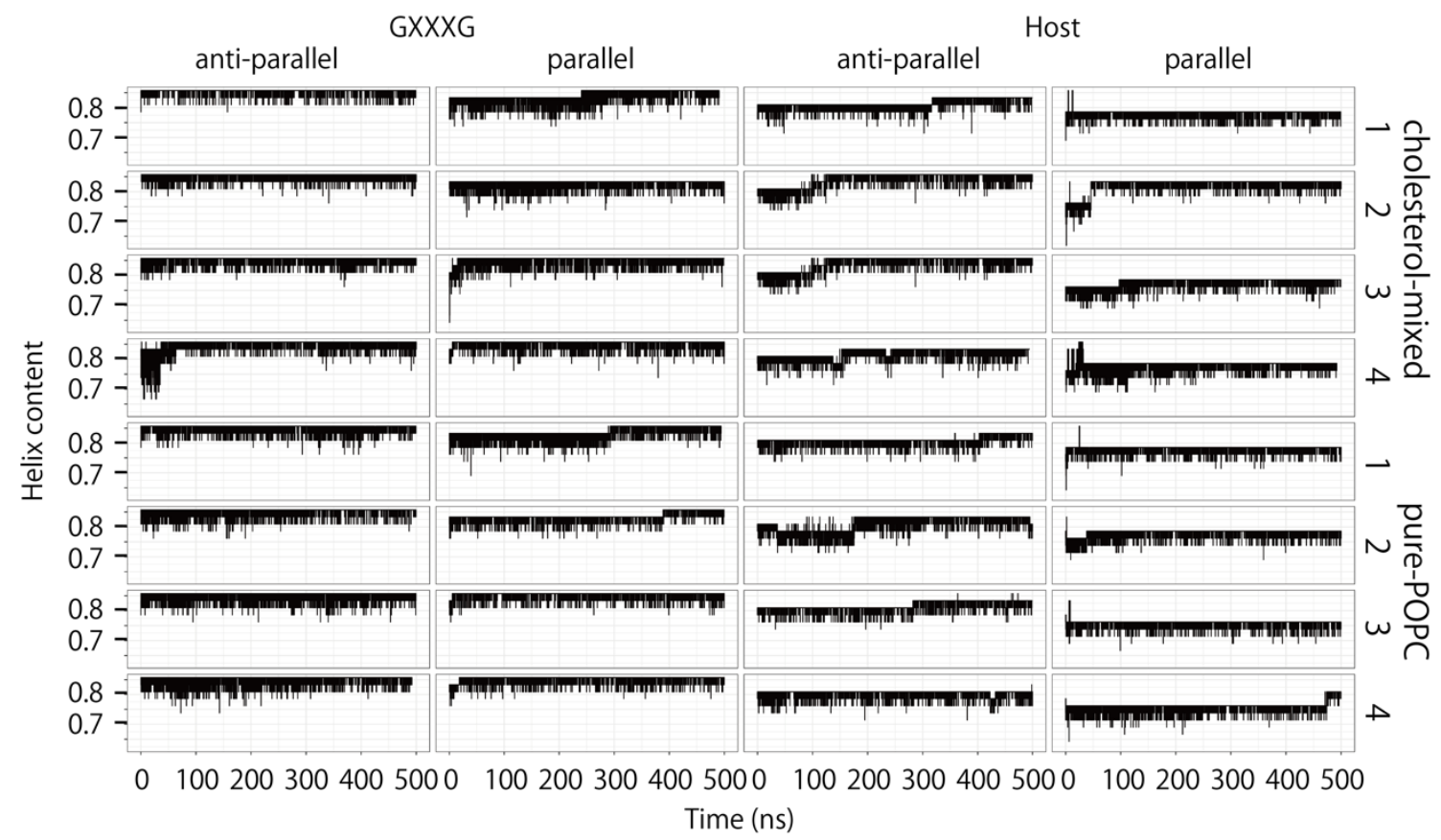

Supplementary Figure S1. Time evolution of helix content of each run. The horizontal and vertical axes indicate time and helix content of two peptides, respectively. The left two columns and right two columns indicate the results of the GXXXG and host peptides, respectively. The first and third columns from the left show the data from the dimer with anti-parallel direction, and others (the second and fourth) show the data from the dimer with parallel direction. The top four rows and bottom four rows are the data from cholesterol-mixed and pure-POPC membranes, respectively. The numbers at the right side of the figure from 1 to 4 correspond to the trajectory 1 to 4 , respectively. 


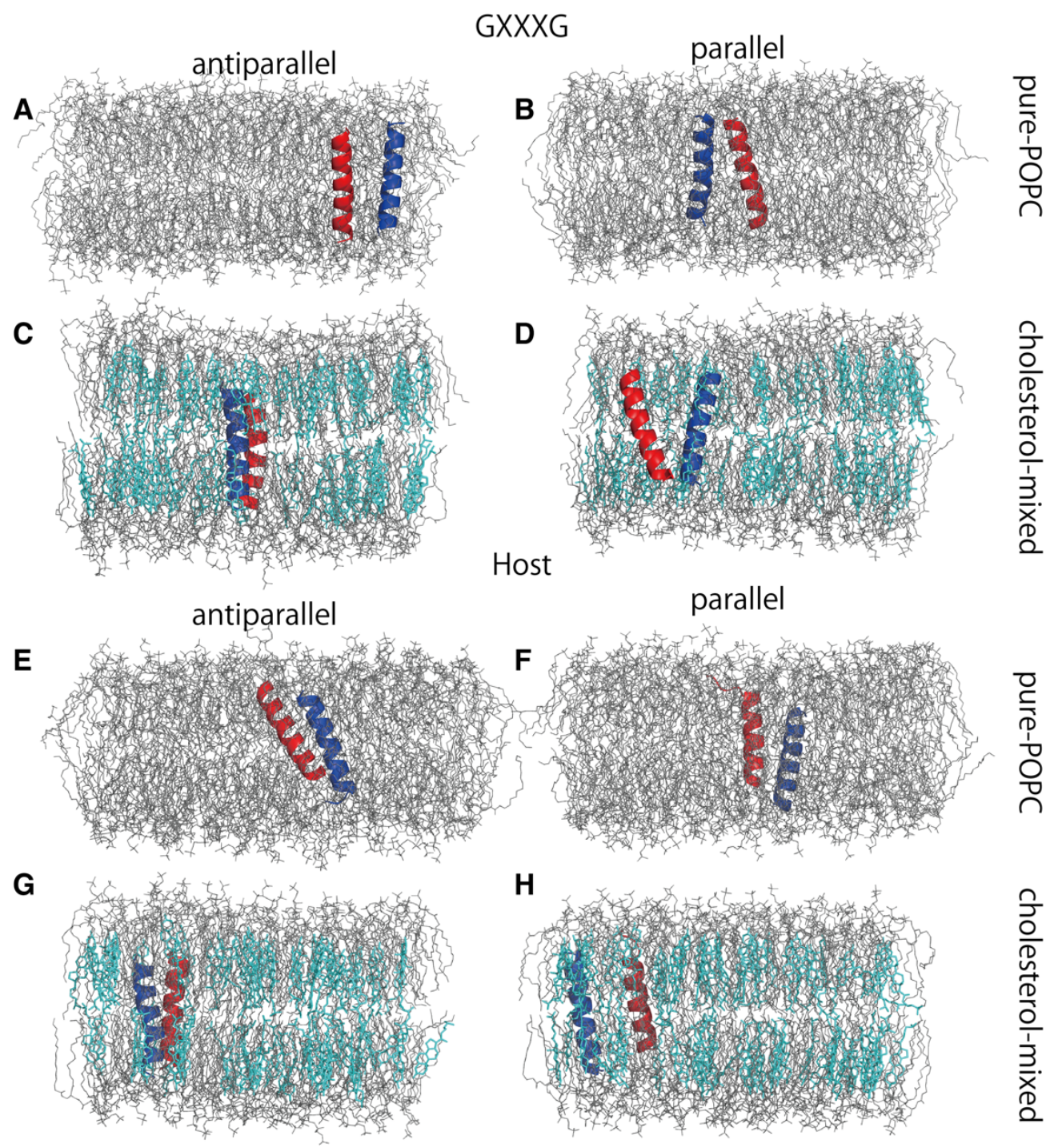

Supplementary Figure S2. Examples of snapshots at the final steps. The top two rows (A, B, C, and D) and bottom two rows (E, F, G, and H) show the structures of the GXXXG and host dimers, respectively. The left $(\mathrm{A}, \mathrm{C}, \mathrm{E}$, and $\mathrm{G})$ and right $(\mathrm{B}, \mathrm{D}, \mathrm{F}$, and $\mathrm{H})$ columns show the dimers in the antiparallel and parallel configurations, respectively. The first and third rows (A, B, E, and F) show the systems with the pure-POPC membrane, and the second and fourth rows (C, D, G, and H) show those with the cholesterol-mixed membrane. See also the legend of Figure 1. 

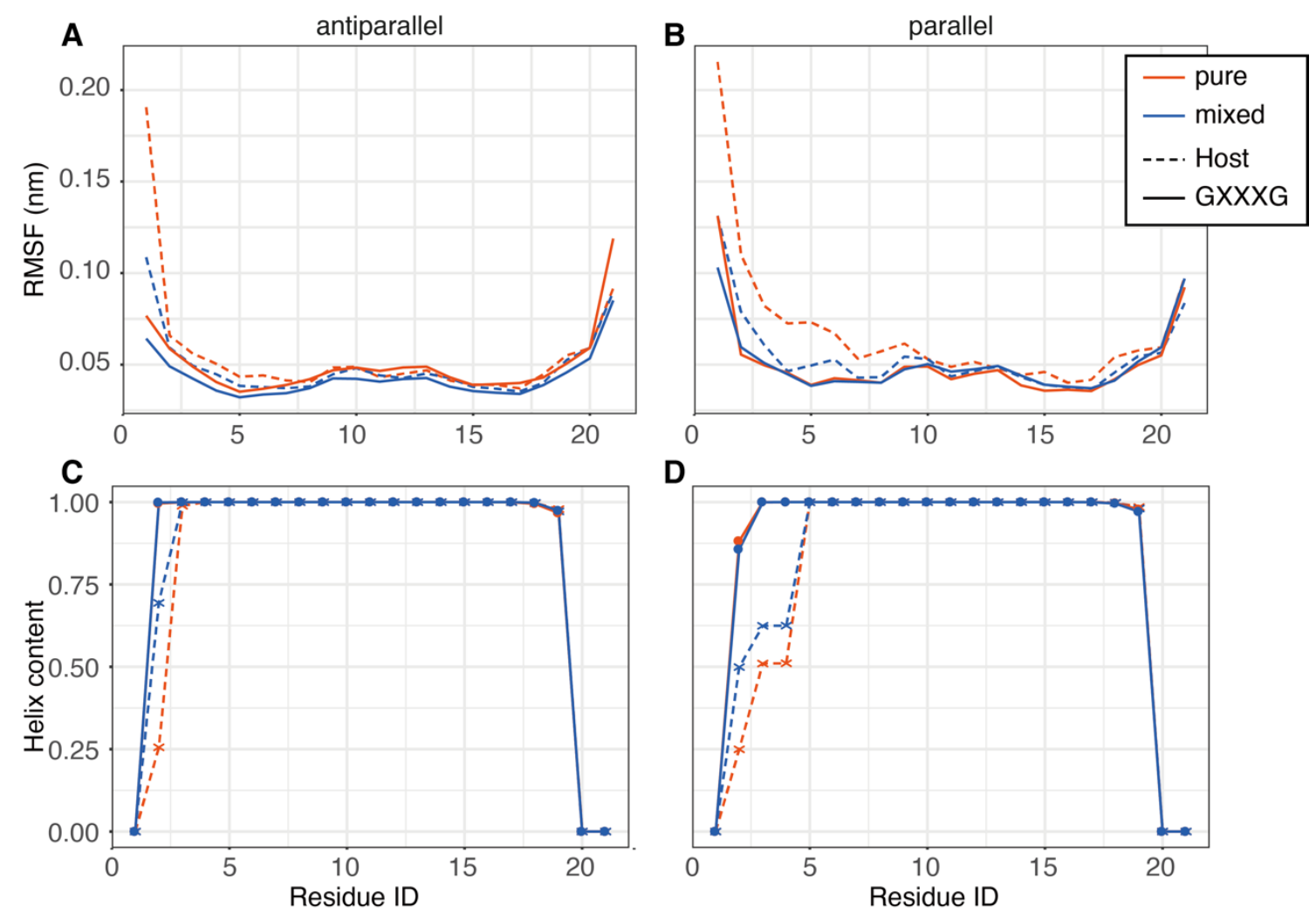

Supplementary Figure S3. (A, B) RMSF and (C, D) helix content of each residue. The red and blue lines indicate the pure-POPC and cholesterol-mixed membrane, respectively. The solid and dashed lines show the GXXXG and host peptides, respectively. (A, C) Antiparallel and (B, D) parallel directions of the dimer. 


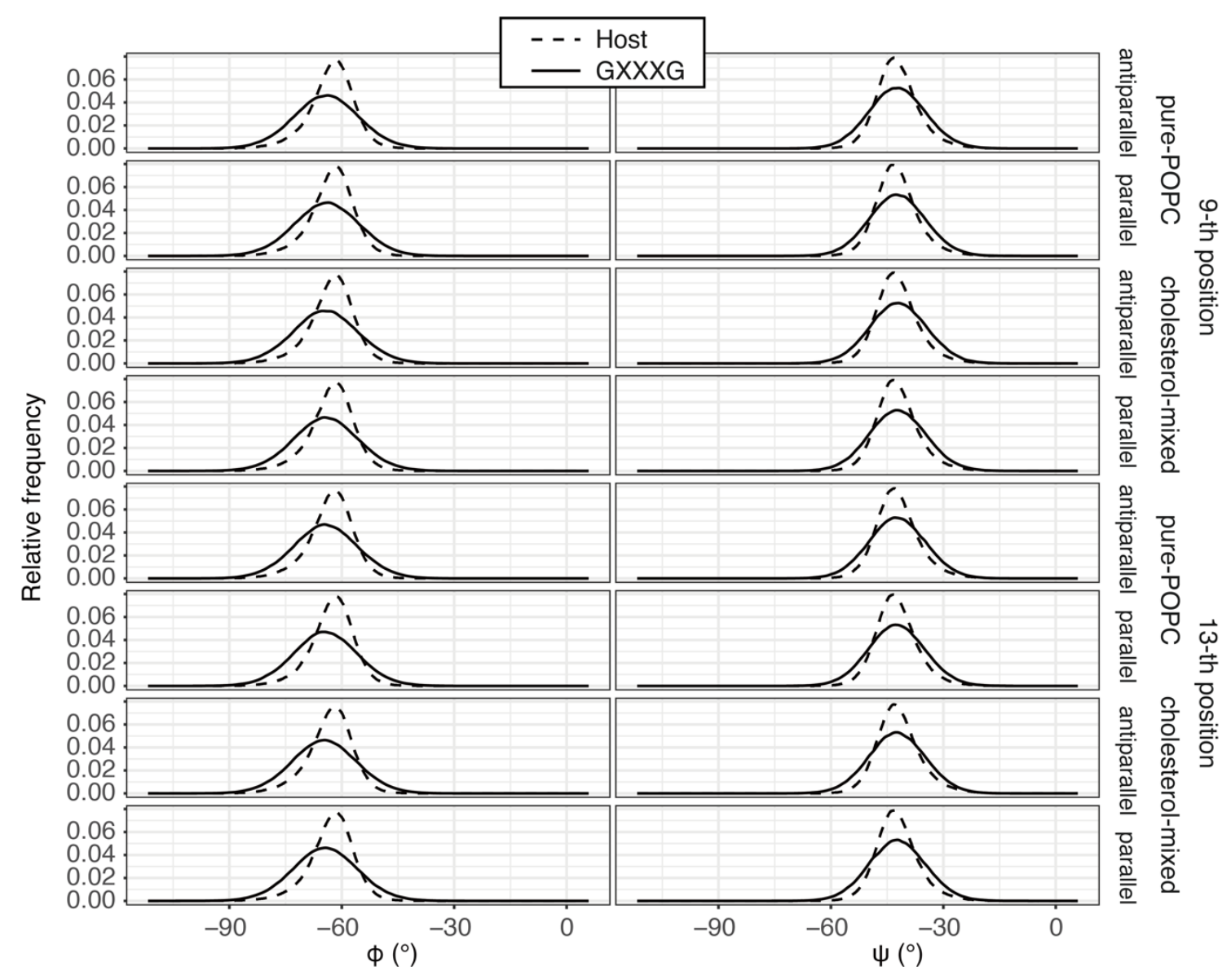

Supplementary Figure S4. The backbone dihedral angles 9-th and 13-th residues, which were mutated into Gly from Ala in the GXXXG peptide. The solid and dashed lines show the GXXXG and host peptides, respectively. The horizontal axis indicates phi (left column) and psi (right column) angles, and the vertical axis indicates relative frequency in the ensemble. Each row shows different conditions of simulation and positions of the residue (9-th or 13-th); see the rightmost part of the figure. 

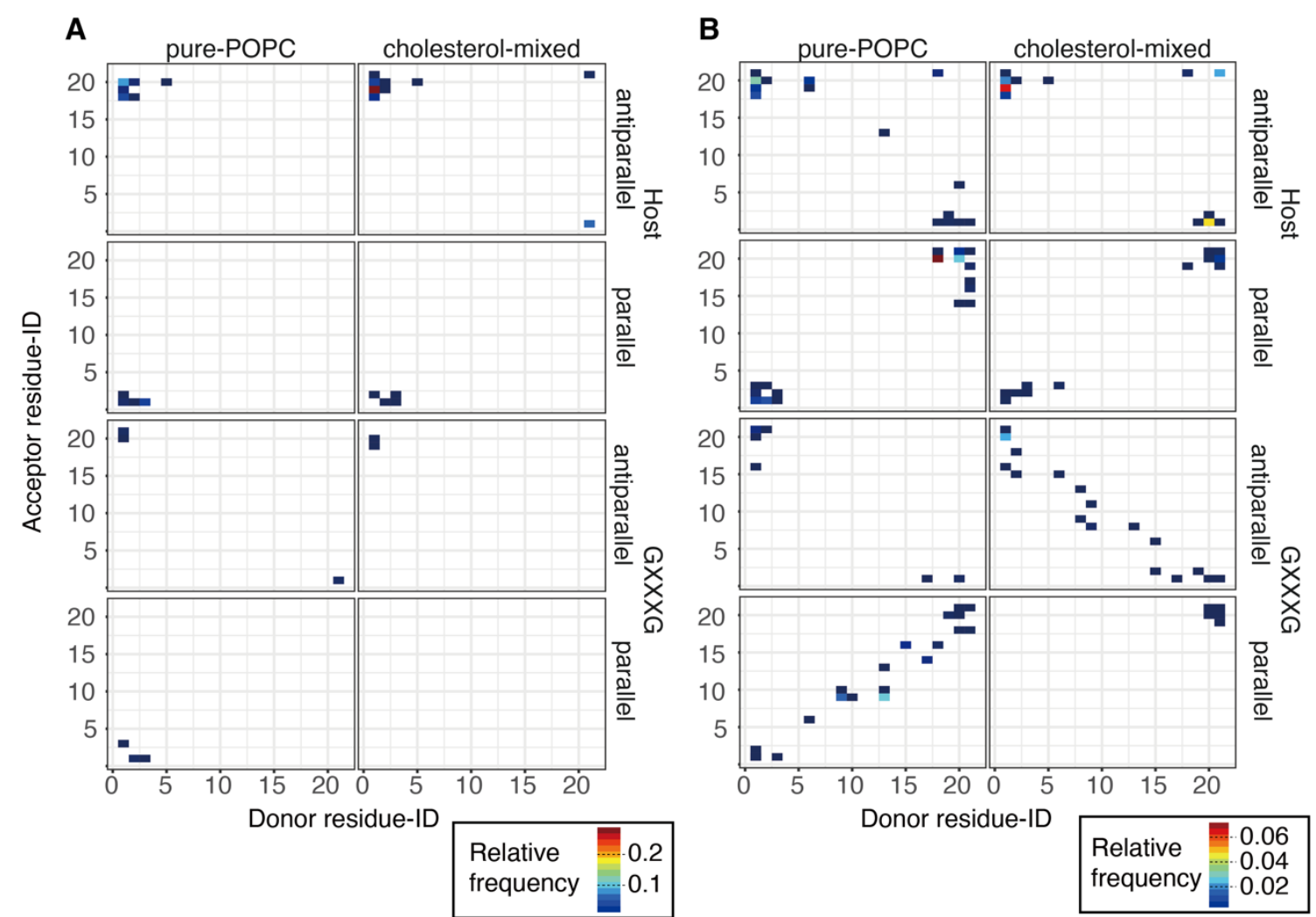

Supplementary Figure S5. Residue-wise frequency for (A) N-H ... O and (B) C $\alpha-\mathrm{H} \ldots$ O hydrogen bond formation. The horizontal and vertical axes indicate the residue number in each peptide. The color means the frequency for hydrogen bonds at the corresponding pair of residues. 


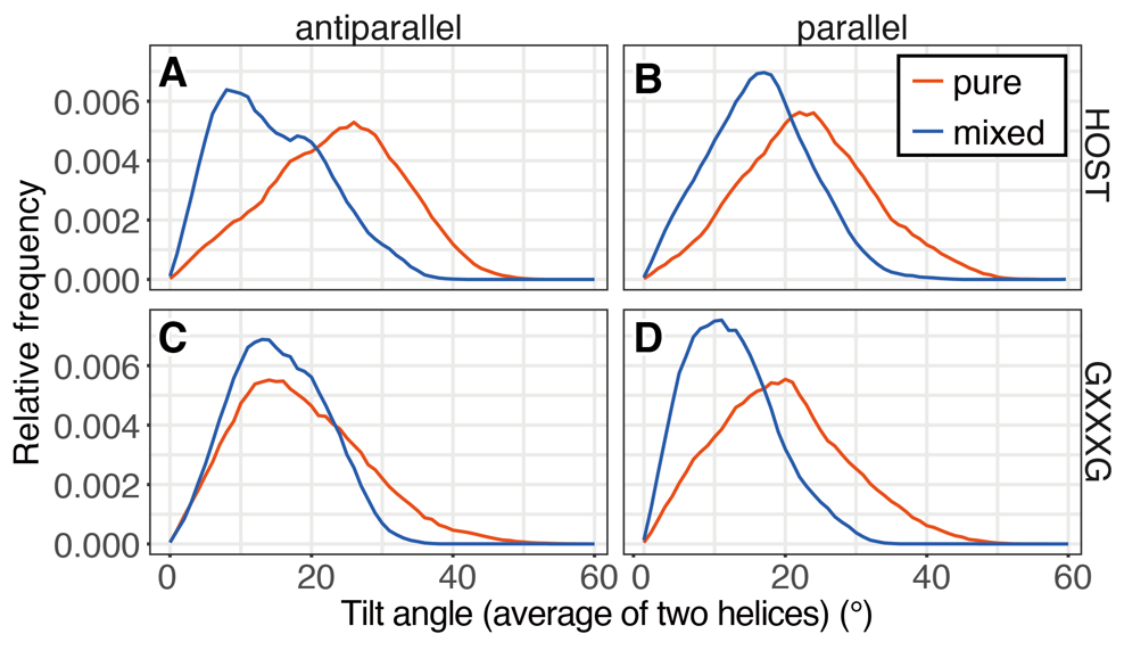

Supplementary Figure S6. Distributions of tilt angles defined as an average of tilt angles of the two peptide helices against the membrane normal. Red and blue lines indicate results of the pure-POPC and cholesterol-mixed membranes, respectively. The upper row (A and B) and lower row (C and D) show the data from the host and GXXXG peptides, respectively. The left column (A and C) and right column (B and D) show the antiparallel and parallel directions of the dimer, respectively. 

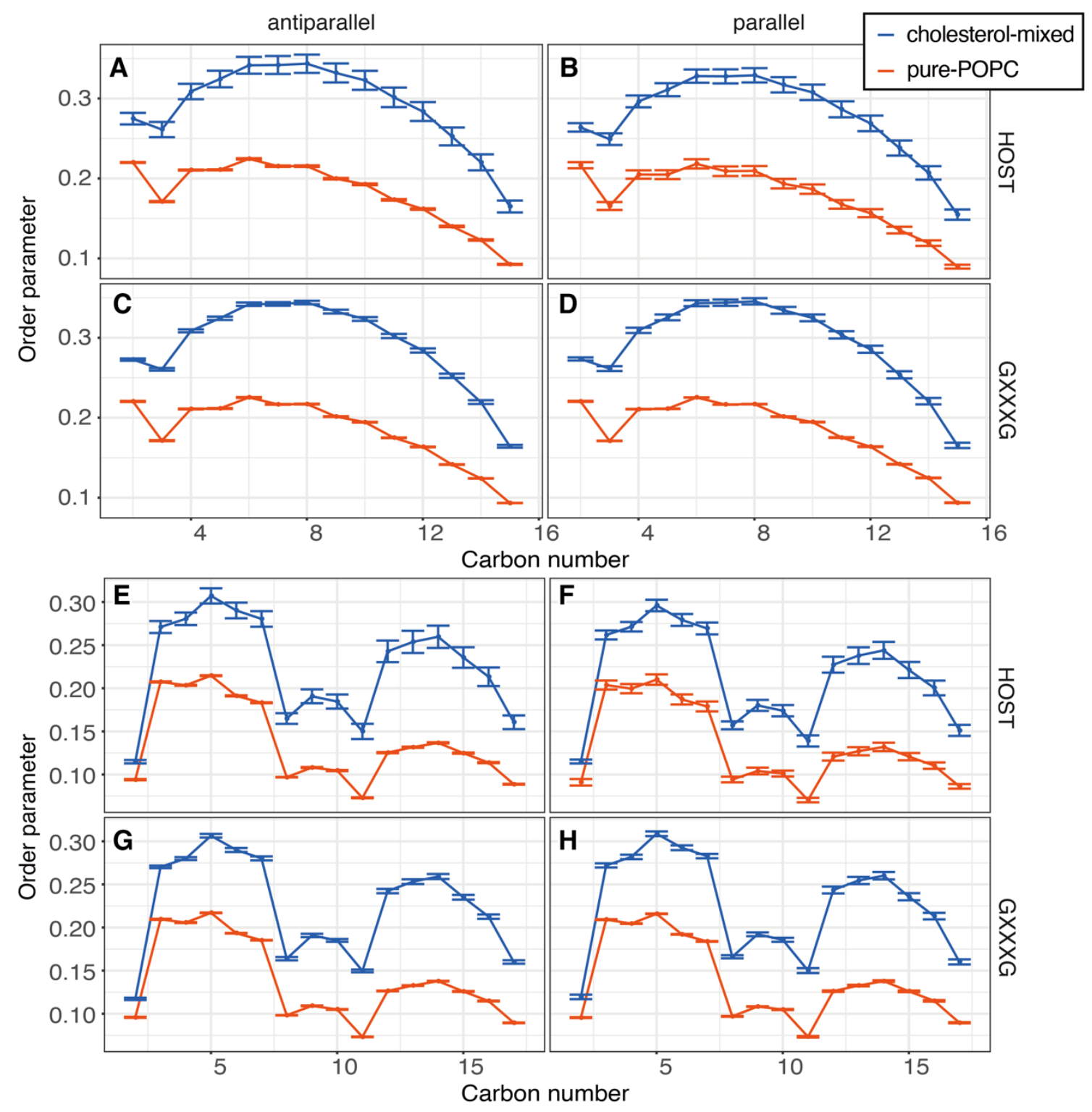

Supplementary Figure S7. Order parameters for (A)-(D) sn-1 and (E)-(H) sn-2 chains of POPC. The order parameters were analyzed by using gmx order module in GROMACS software package. Error bars indicate the standard errors over the four trajectories. 


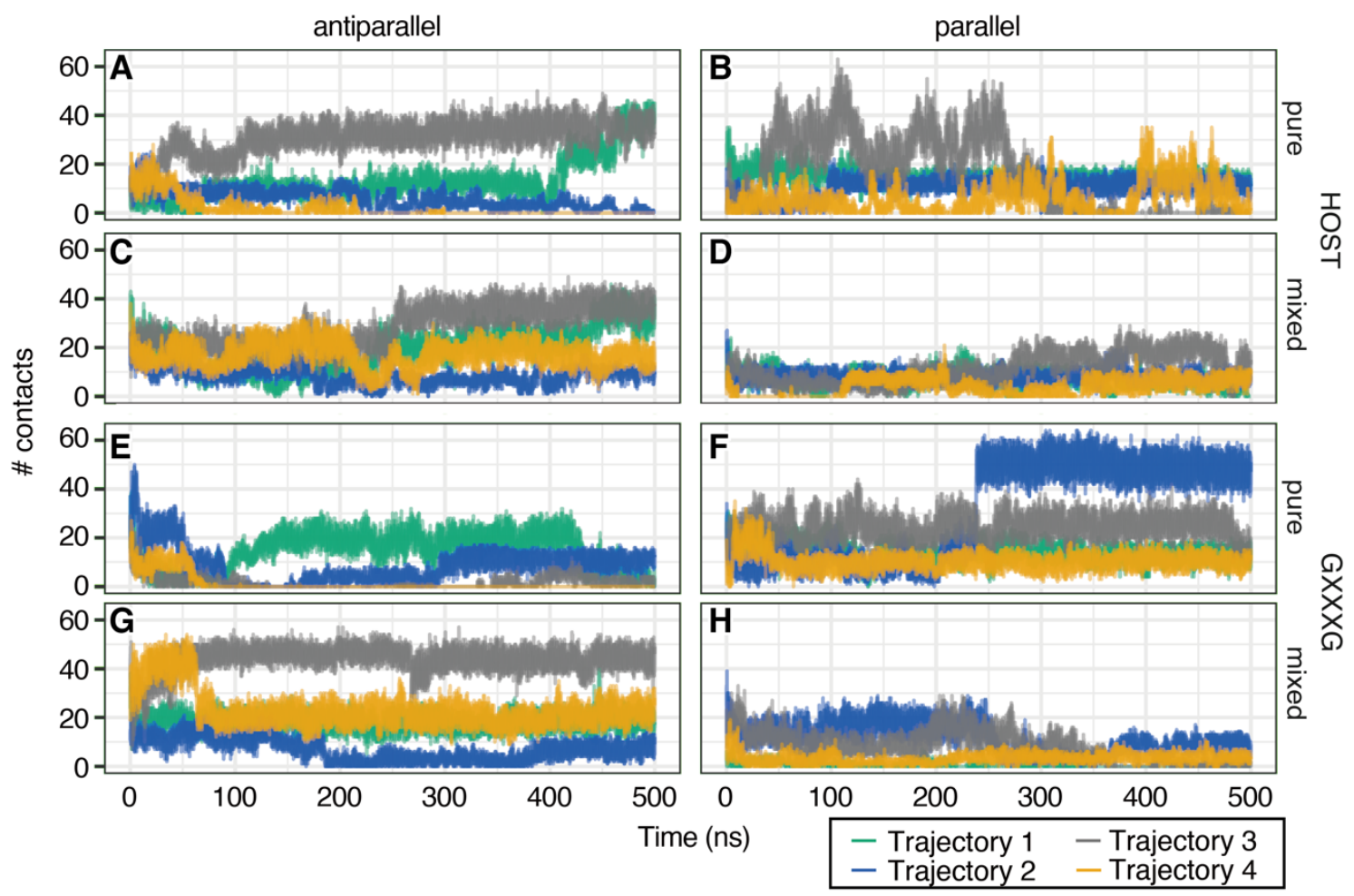

Supplementary Figure S8. Time evolution of the number of inter-peptide contacts in each replicate of simulations. The left (A, C, E, and G) and right (B, D, F, and H) columns show the data from the dimers in the antiparallel and parallel configurations, respectively. The first and third rows (A, B, E, and F) present the systems with the pure-POPC membrane, and the second and fourth rows $(\mathrm{C}, \mathrm{D}, \mathrm{G}$, and $\mathrm{H}$ ) present those with the cholesterol-mixed membrane. The top two rows (A, B, C, and D) and the bottom two rows (E, F, G, and $\mathrm{H}$ ) present results of the host and GXXXG peptides, respectively. 


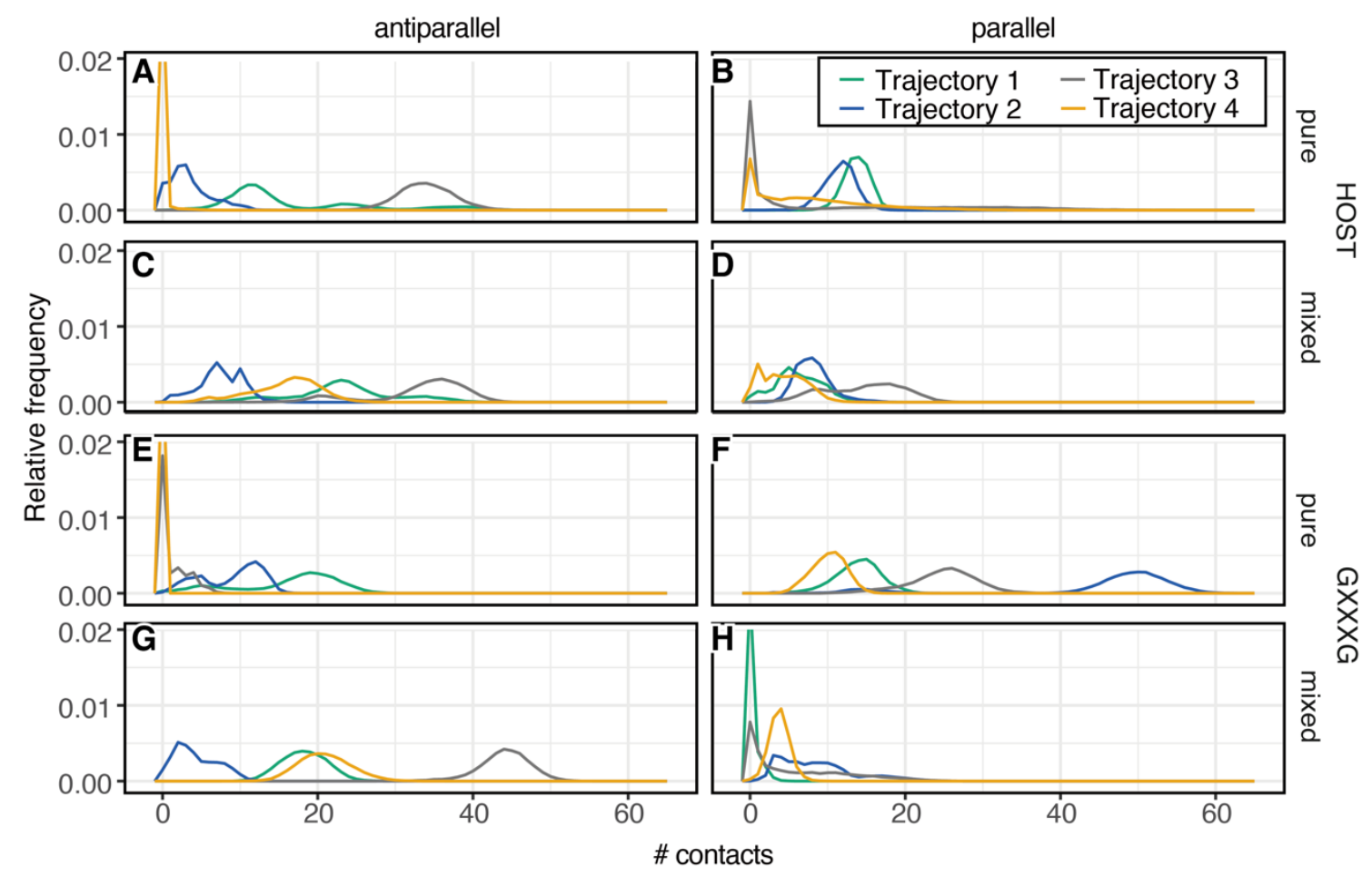

Supplementary Figure S9. Distribution of the number of inter-peptide contacts in each replicate of simulations. The left (A, C, E, and $\mathrm{G})$ and right $(\mathrm{B}, \mathrm{D}, \mathrm{F}$, and $\mathrm{H})$ columns show the data from the dimers in the antiparallel and parallel configurations, respectively. The first and third rows (A, B, E, and F) present the systems with the pure-POPC membrane, and the second and fourth rows $(\mathrm{C}, \mathrm{D}, \mathrm{G}$, and $\mathrm{H}$ ) present those with the cholesterol-mixed membrane. The top two rows (A, B, C, and D) and the bottom two rows (E, F, G, and $\mathrm{H})$ present results of the host and GXXXG peptides, respectively. 


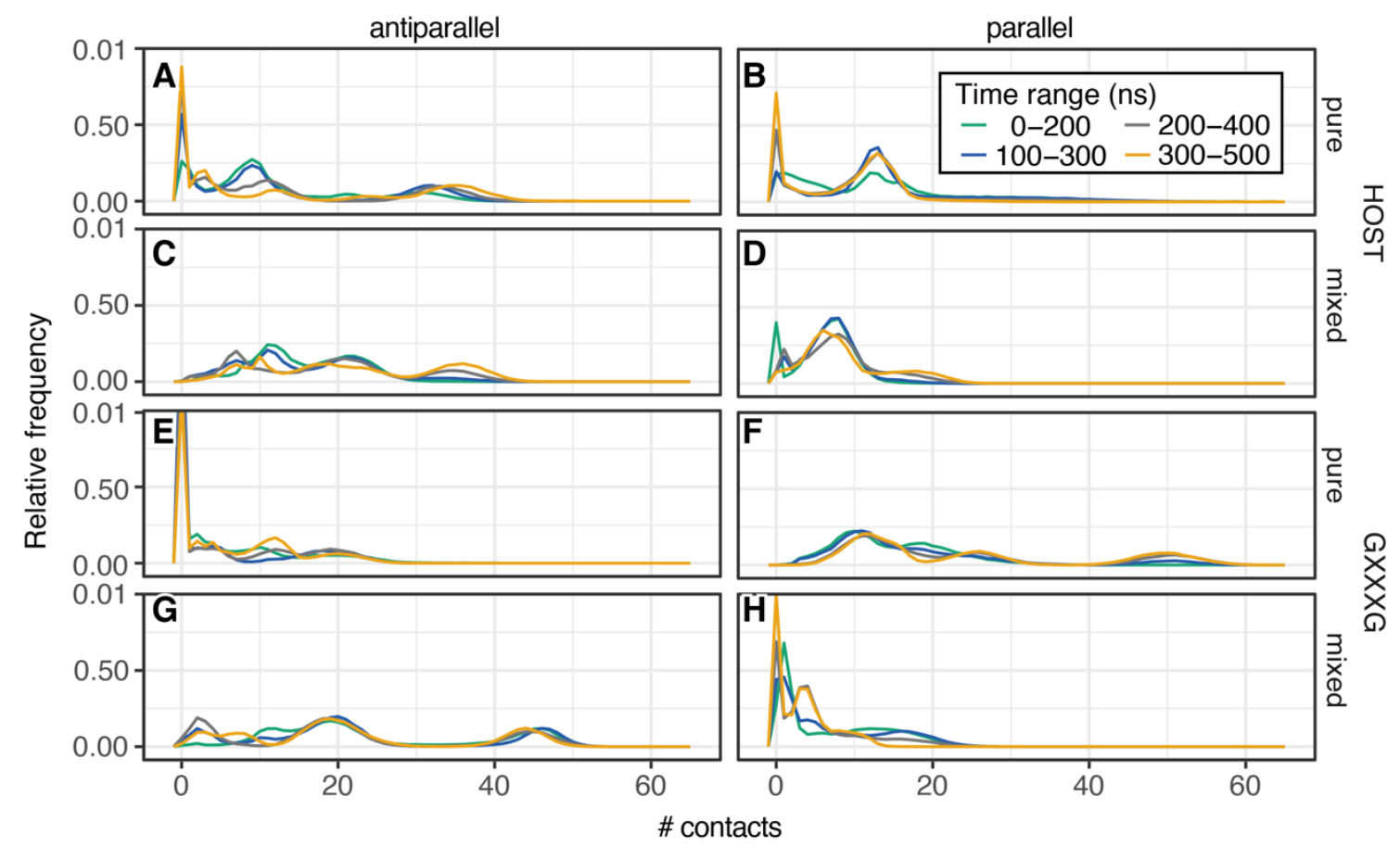

Supplementary Figure S10. Distribution of the number of inter-peptide contacts in each 200-ns time window. The left (A, C, E, and $\mathrm{G}$ ) and right (B, D, F, and $\mathrm{H}$ ) columns show the data from the dimers in the antiparallel and parallel configurations, respectively. The first and third rows (A, B, E, and F) present the systems with the pure-POPC membrane, and the second and fourth rows (C, D, G, and H) present those with the cholesterol-mixed membrane. The top two rows (A, B, C, and D) and the bottom two rows $(\mathrm{E}, \mathrm{F}, \mathrm{G}$, and $\mathrm{H})$ present results of the host and GXXXG peptides, respectively. 


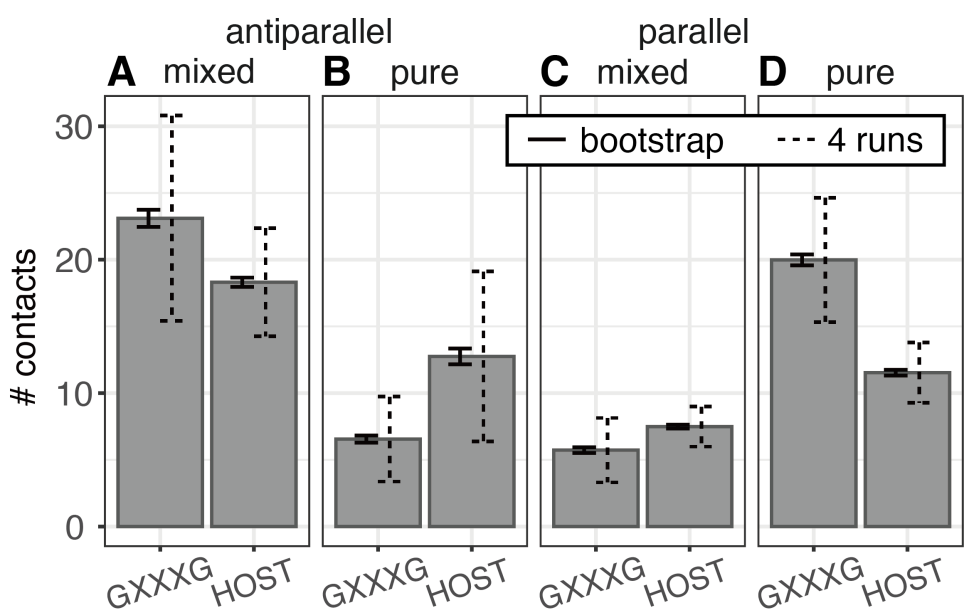

Supplementary Figure S11. Average and errors for number of inter-residue contacts. (A and C) and (B and D) show the data for cholesterol-mixed and pure-POPC membranes, respectively. (A and B) and $(\mathrm{C}$ and $\mathrm{D})$ present the antiparallel and parallel directions of the dimer, respectively. The error bars indicate the standard errors over the four trajectories (dashed line) and the standard errors calculated with the bootstrap analysis in the same manner as Figure 3. 
Supplementary Table S1. Summary of simulation systems

\begin{tabular}{cccccccc}
\hline Membrane & peptide & direction & \# atoms & XY (nm) & Z (nm) & \multicolumn{2}{c}{$\begin{array}{c}\text { Simulation \# runs } \\
\text { length (ns) }\end{array}$} \\
\hline pure-POPC & host & parallel & 66,040 & 9.47 & 8.00 & 500 & 4 \\
pure-POPC & host & anti-parallel & 65,878 & 9.47 & 8.00 & 500 & 4 \\
pure-POPC & GXXXG & parallel & 61,592 & 9.47 & 7.49 & 500 & 4 \\
pure-POPC & GXXXG & anti-parallel & 61,379 & 9.47 & 7.45 & 500 & 4 \\
cholesterol-mixed & host & parallel & $58,167 \sim 58,245^{\dagger}$ & 8.89 & 8.00 & 500 & 4 \\
cholesterol-mixed & host & anti-parallel & $58,098 \sim 58,179^{\dagger}$ & 8.89 & 8.00 & 500 & 4 \\
cholesterol-mixed & GXXXG & parallel & $54,283 \sim 54,373^{\dagger}$ & 8.89 & 7.49 & 500 & 4 \\
cholesterol-mixed & GXXXG & anti-parallel & $53,929 \sim 54,136^{\dagger}$ & 8.89 & 7.45 & 500 & 4 \\
\hline
\end{tabular}

${ }^{\dagger}$ For the simulations with cholesterol-mixed membrane, different initial structures were generated among four runs of simulations. 